\title{
Validity of the Linear Growth Equation for Interface Evolution for Copper Electrodeposition in the Presence of Organic Additives
}

\author{
L. Vázquez,* R. C. Salvarezza, and A. J. Arvia \\ Instituto de Investigaciones Fisicoquímicas Teóicas Y Aplicadas (INIFTA), Sucursal 4, \\ Casilla de Correo 16, (1900) La Plata, Argentina
}

(Received 3 March 1997)

\begin{abstract}
The interface evolution of copper electrodeposits produced on copper from a thiorea-derivativecontaining acid plating bath at a low current density and $298 \mathrm{~K}$ was studied by atomic force microscopy (AFM). The dynamic scaling theory was applied to AFM images leading to scaling exponents, which are consistent with the predictions of the complete linear equation for interface growth. In agreement with the theory, experimental data show, for the interface evolution, a crossover from a surface diffusion controlled regime to an Edwards-Wilkinson regime as the length scale increases. [S0031-9007(97)03581-3]
\end{abstract}

PACS numbers: 68.55.Jk, 81.10.Bk, 82.20.Wt

Metal electrodeposition is one of the methods most commonly used for film preparation. This process has been extensively studied in relation to fractal patterns grown at high electrodeposition rates for different metals and environments [1]. In contrast, little is known about electrodeposition patterns grown at low rates. It has been recently found that the interface evolution of copper electrodeposits grown at low rate becomes unstable [2,3] turning the Edwards-Wilkinson (EW) [4] and KardarParisi-Zhang [5] stochastic equations inapplicable. It has also been found that the addition of organic additives to the plating bath suppresses unstable growth and reduces the roughness of electrodeposits [6]. Organic additives are widely used in metal electroplating to improve coating quality, although their role is not fully understood yet, a matter which is relevant for technological applications. Recently a great effort has been made in the understanding of copper electrodeposition and the role of organic additives on the interface evolution [7-9].

The scaling properties of the interface evolution are usually studied through the dynamic scaling theory $[10,11]$ applied to surface profiles. According to this theory, for a surface profile of length $L$ consisting of $N$ points of average height $h$, when $t \rightarrow 0$ the interface width $W(L, h)$ scales as [10]

$$
W(t) \propto t^{\beta}
$$

and, when $t \rightarrow \infty$, it scales as

$$
W(L, t) \propto L^{\alpha},
$$

where $W(L, t)$ is defined by

$$
W(L, t)=\left[(1 / N) \Sigma\left(h_{i}-h\right)^{2}\right]^{1 / 2},
$$

and $h_{i}$ is the deposit height measured along the $x$ direction at the point $x_{i}, \beta$, and $\alpha$ are the growth and roughness exponents, respectively, and the dynamic exponent is $z=\alpha / \beta$.
Several stochastic partial differential equations have been proposed to describe the evolution of rough interfaces [11]. In the absence of nonlinear terms determining the scaling properties, a general linear equation has been proposed for the interface growth of solid films [12]:

$$
\partial h / \partial t=\nu \nabla^{2} h-K \nabla^{4} h+\eta,
$$

where the first and second terms of the right-hand side of Eq. (4) describe the surface relaxation by "surface tension" through adsorption/desorption, and surface diffusion, respectively, and $\eta$ is the stochastic noise ( $\nu, K$ are constants). Equation (4) predicts the existence of two distinct phases, each one with a different morphology and scaling exponents $\alpha$ and $\beta$. These phases are delimited by the crossover length $L^{*}=(K / \nu)^{1 / 2}$ [11]. For $L<L^{*}$, the diffusive term dominates and Eq. (4) [13] reduces to

$$
\partial h / \partial t=-K \nabla^{4} h+\eta,
$$

which leads to $\alpha=1$ and $\beta=0.25$ in three dimensions (3D) [13]. On the other hand, for $L \rightarrow \infty$, the adsorption/ desorption term governs the scaling properties so that the Edwards-Wilkinson [4] interface evolution equation is obtained:

$$
\partial h / \partial t=\nu \nabla^{2} h+\eta .
$$

Equation (6) predicts $\alpha=0$ and $\beta=0$ in three dimensions. In this case, the correlations decay logarithmically so that, for $1 \ll h \ll L \quad(t \rightarrow 0)$, it is expected $W \propto(K / \nu)(\log \nu t)^{0.5}$ and, for $h \gg L \gg 1$, $W \propto(K / \nu)(\log L)^{0.5}$ [4]. Despite the fact that there are several experimental systems [14] that obey Eq. (5), and Eq. (6) has recently been successfully applied to sedimentation processes [15], the validity of Eq. (4) has not yet been verified.

In this Letter we report a study on the interface evolution during copper electrodeposition from additivecontaining acid plating baths. The validity of Eq. (4), 
covering from a diffusion-controlled interface regime to the EW behavior through a crossover region, was verified.

The electrodeposition of copper was carried out in a three dimensional glassmade electrochemical cell under galvanostatic conditions using an annealed polycrystalline $\mathrm{Cu}$ cathode $\left(1 \mathrm{~cm}^{2}\right.$ area, root mean square saturated roughness $4 \mathrm{~nm}$ ) as working electrode, a large Pt plane $\left(4 \mathrm{~cm}^{2}\right)$ as counterelectrode, and a saturated calomel electrode as reference. An aqueous solution containing $150 \mathrm{~g} / 1 \mathrm{CuSO}_{4.5} \mathrm{H}_{2} \mathrm{O}+50 \mathrm{~g} / 1 \mathrm{H}_{2} \mathrm{SO}_{4}+x \mathrm{mM}$ 1,3-diethyl-2-thiourea $(0.3 \leq x \leq 0.4)$ saturated with purified $\mathrm{N}_{2}$ was used for $\mathrm{Cu}$ electrodeposition. Still plating baths were employed at $298 \mathrm{~K}$. Copper electrodeposition was performed at $j=0.02 \mathrm{~A} \mathrm{~cm}^{-2}$, an apparent current density lower than the limiting current density calculated from the free-convective diffusion mass transport equation [16]. The irreversible behavior of the polarization curve run at $0.001 \mathrm{~V} \mathrm{~s}^{-1}$ in the working solution, and the independence of its overvoltage-dependent portion on stirring indicated that the electrochemical reaction is under activation control. The electrodeposition time was in the range $60 \leq t \leq 3600 \mathrm{~s}$. For this range of $t$ and $j=0.02 \mathrm{~A} \mathrm{~cm}^{-2}$ the average film thickness $h$ was in the range $5 \times 10^{-5} \mathrm{~cm} \leq h \leq 3 \times 10^{-3} \mathrm{~cm}$. The topography of the electrodeposits surface was ex situ imaged immediately after copper electrodeposition using a Nanoscope III atomic force microscope (AFM), operating in the contact mode at ambient conditions. Silicon nitride cantilevers were used. Images were taken and analyzed using the single image dynamic scaling method after fitting the instrument plane and applying a subtracting procedure $[17,18]$. Times required for AFM imaging were always smaller than $60 \mathrm{~s}$. Typical diffusion lengths for fcc metal covered by sulfurcontaining organic molecules are smaller than $1 \mathrm{~nm} / \mathrm{min}$, as, under these conditions, the surface diffusion coefficient of metal atoms at $298 \mathrm{~K}$ is close to $10^{-17} \mathrm{~cm}^{2} \mathrm{~s}^{-1}$ [19]. Therefore, the use of ex situ AFM imaging for scaling at lengths greater than $1 \mathrm{~nm}$, and measuring times involved in this work is justified.

AFM images of $\mathrm{Cu}$ electrodeposits produced from the plating bath reveal the presence of crystals of average size $d$ with a smooth surface. It is observed that $d$ decreases as $x$ is increased from 0.3 to 0.4 [Figs. 1(a), 1(b)]. Cross sections of the AFM images demonstrate that, at early times, the initially planar interface becomes increasingly irregular (Fig. 2). At late times, the interface reaches a steady state roughness from the plot of $W_{\mathrm{AFM}}$ vs $t$, it is evident that $W_{\mathrm{AFM}}$ increases slowly with the electrodeposition time reaching saturation for $t>t_{s}, t_{s}$ being a $x$ dependent value (Fig. 3).

After the steady state has been reached, the interface width is length scale dependent obeying the proportionality

$$
W_{\mathrm{AFM}}\left(t \gg t_{s}\right) \propto L^{\alpha},
$$

where $L$ is the length of a segment of a AFM scan of size $S(S>L)$ taken in the fast AFM $x$ direction. (a)

(b)
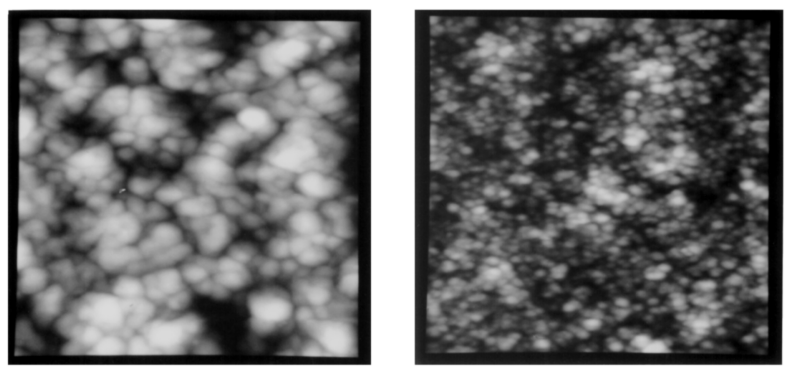

FIG. 1. $2 \times 2 \mu \mathrm{m}^{2}$ AFM images of $\mathrm{Cu}$ electrodeposits produced from the additive-containing plating bath at $j=0.02 \mathrm{~A} \mathrm{~cm}^{-2}$ and $298 \mathrm{~K}$ for $t=360 \mathrm{~s}$. (a) $x=0.3$ and (b) $x=0.4$.

The $W_{\mathrm{AFM}}$ vs $L$ logarithmic plots from AFM images of copper films for $0.3 \leq x \leq 0.4$ [Fig. 4(a)] show three distinguishable regions, namely, a linear region (I) for $L<d$ with slope $\alpha(I)=0.90 \pm 0.05$, a crossover region (region II) for $d \leq L \leq L_{s}$, and region III for $L>L_{s}$, where $W_{\mathrm{AFM}}$ increases very slowly with $L(\alpha \approx 0)$. The first and third regimes define a crossing point $L^{*}$. The values of $W_{\mathrm{AFM}}, d$, and $L^{*}$ decrease on increasing $x$ from 0.3 to 0.4 . For region III, the $\left(W_{\mathrm{AFM}}\right)^{2}$ vs $\log L$ plots show a linear behavior [Fig. 4(b)]. The crossover region is delimited by the average crystal size $d$ and the average size of the largest irregularities $L_{s}$ [Fig. 4(c)].

For region I the value $\beta=0.25 \pm 0.05$ was obtained from the time evolution of $W_{d}$, and the value of $W_{\mathrm{AFM}}$ was measured at $L=d$ (Fig. 5). Thus, the exponents $\alpha=0.9$ and $\beta=0.25$ are close to those expected from the WolfVillain model [Eq. (5)], where surface diffusion operates. For region III, the roughening kinetics was determined from the $W_{\mathrm{AFM}} \mathrm{vs} t$ data (Fig. 3) for $L>L_{s}$ and $t<t_{s}$. In this case, $W_{\mathrm{AFM}}$ obeys a $\left(W_{\mathrm{AFM}}\right)^{2}$ vs $\log t$ relationship (Fig. 3 inset). For $t>t_{s}, W_{\mathrm{AFM}}$ becomes independent of $t$ so that $\beta=0$, i.e., the long time behavior of the EW model is observed.

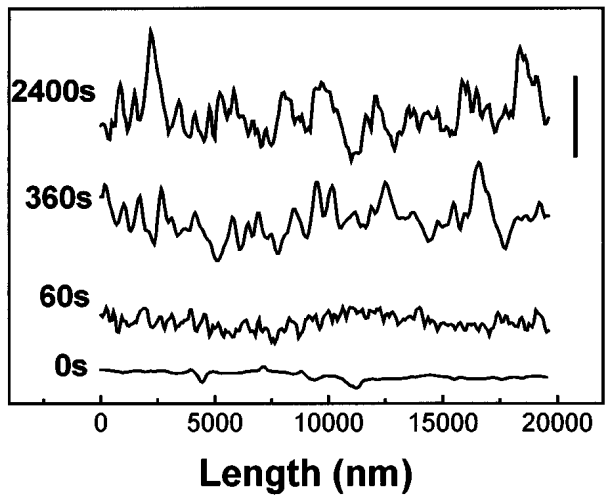

FIG. 2. AFM profiles from $20 \times 20 \mu \mathrm{m}^{2}$ AFM images of copper electrodeposits produced in an additive-containing plating bath with $x=0.3$ at different times. The bar indicates $200 \mathrm{~nm}$. 


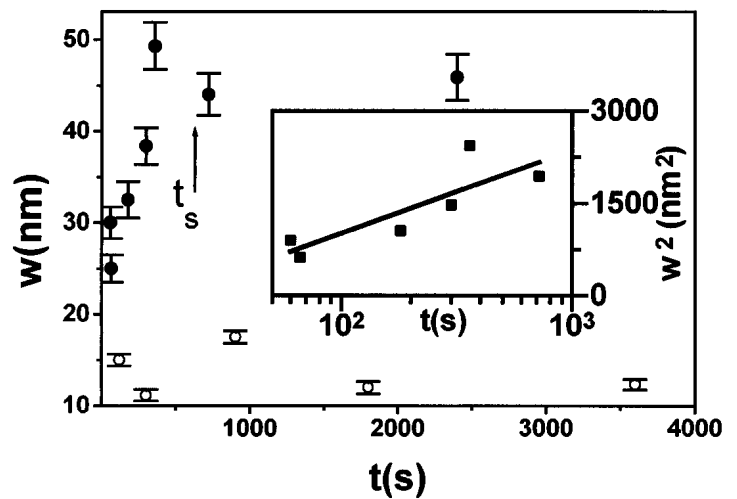

FIG. 3. $W$ vs $t$ plots derived from $20 \times 20 \mu \mathrm{m}^{2}$ AFM images of copper electrodeposits produced from additivecontaining plating baths with $x=0.3(\bullet)$ and $x=0.4(\circ)$. The arrow indicates the saturation time $t_{s}$ for $x=0.3$. Inset: $W^{2}$ vs $t$ semilogarithmic plot corresponding to data of the main part of the figure for $x=0.3$ and $t<t_{s}$. The continuous line indicates the best $W^{2} \propto \log t$ fitting for $t<t_{s}$. Data errors are smaller than the symbol size.

Furthermore, the validity of the EW equation should imply a change in roughness proportional to the square of the crossover length ratio [20]. In fact, data shown in Figs. 3 and 4 fulfill the relationship $W_{\mathrm{AFM}}(x=0.3) / W_{\mathrm{AFM}}(x=$ $0.4) \approx\left[L^{*}(x=0.3) / L^{*}(x=0.4)\right]^{2}$. Figure 3 also indicates that the crossover time $t_{c}$ from the diffusive to EW regime should be less than $60 \mathrm{~s}$ as only the logarithmic dependence was observed in the sampling time range. The saturation time $t_{s}$ and the saturation length $L_{s}$ can be related through the equation $L_{s}=\left(2 \nu t_{s}\right)^{1 / 2}$, which allowed us to estimate the value of $\nu$ [20]. Thus, for $x=0.3$, and using $L_{s}=3 \times 10^{-4} \mathrm{~cm}$ and $t_{s}=720 \mathrm{~s}$, it results in $\nu \approx 10^{-10} \mathrm{~cm}^{2} \mathrm{~s}^{-1}$. From this figure, the crossover length $L^{*}=6 \times 10^{-5} \mathrm{~cm}$, and the relationship $L^{*}=(K / \nu)^{1 / 2}$ [21], it results in $K \approx 10^{-19} \mathrm{~cm}^{4} \mathrm{~s}^{-1}$. This figure is only 1 order of magnitude higher than that reported for semiconductor surfaces $K=2.8 \times 10^{-20} \mathrm{~cm}^{4} \mathrm{~s}^{-1}$ [21]. By introducing the values of $K$ and $\nu$ in the equation $t_{c}=K / \nu^{2}$ [21], it follows that $t_{c}<60 \mathrm{~s}$, confirming that, in our experiment, the crossover time cannot be observed.

The values $\alpha=0.90$ and $\beta=0.25$ reported in this Letter for $L<d$, i.e., length scales which correspond to the crystal surface, are in agreement with the expectations of Eq. (5). On the other hand, for $L>L_{s}$ and $t \rightarrow t_{s}$, a logarithmic dependence for the interface width with $L$ and $t$ is obtained in agreement with Eq. (6). This fact points out that Eq. (4) is obeyed in the overall time and length scale range for copper electrodeposition from additive-containing acid plating baths. It means that, for lengths smaller than the crystal size, copper adatoms diffusion is driven by local chemical potential gradients due to curvature effects [11]. The contribution of surface diffusion results in smooth grain surfaces. The fact that $d$ decreases as $x$ is increased indicates that the diffusion length of copper adatoms has been reduced by increasing the copper surface coverage by additive molecules as observed for other metals in aqueous solutions $[9,19,22]$. This decrease in the diffusion length also implies a decrease in both $K$ and $L^{*}$. Thus, the EW
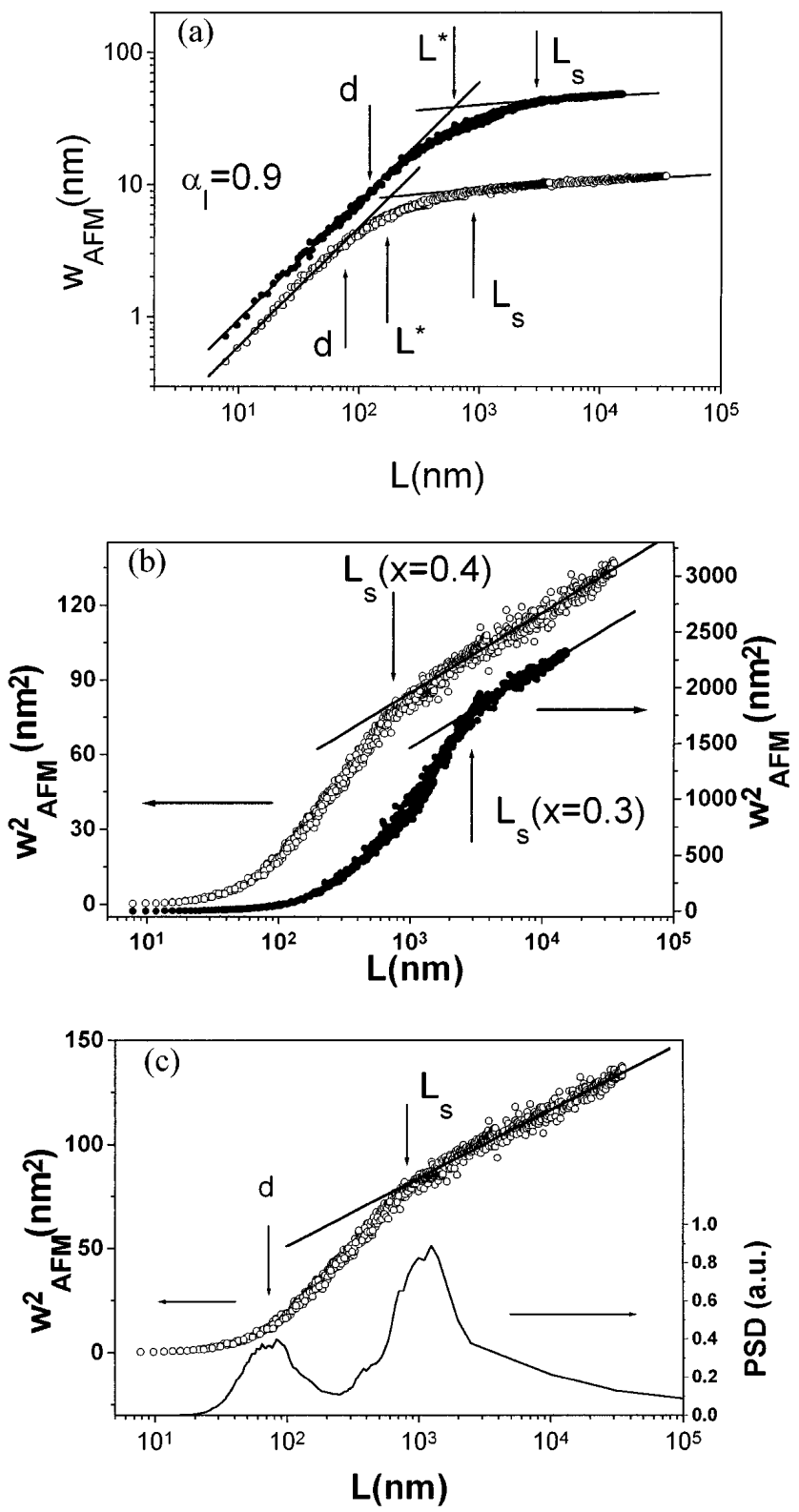

FIG. 4. (a) $W_{\mathrm{AFM}}$ vs $L$ logarithmic plots from the single image dynamic scaling analysis of AFM images of copper electrodeposits produced from additive-containing acid plating baths at $j=0.02 \mathrm{~A} \mathrm{~cm}^{-2}$ to $t=2400 \mathrm{~s}$ and $298 \mathrm{~K}$. (•) $x=$ 0.3 and (०) $x=0.4$. The straight lines for low $L$ values indicate the best $W \propto L^{\alpha}$ fittings for both curves, with a slope $\alpha=0.90$. The continuous lines for large $L$ values indicate the EW region for both curves. Values of $d, L^{*}$, and $L_{s}$ for each curve are marked by arrows. (b) $\left(W_{\mathrm{AFM}}\right)^{2}$ vs $L$ semilogarithmic plot of data shown in (a) (•) $x=0.3$ (right $y$-axis), (o) $x=0.4$ (left $y$-axis). Continuous lines indicate the best $\left(W_{\mathrm{AFM}}\right)^{2} \propto \log L$ fitting for $L>L_{s}$. (c) $\left(W_{\mathrm{AFM}}\right)^{2}$ vs $L$ semilogarithmic plot (left $y$-axis, top curve) and power spectral density (PSD) vs $L$ semilogarithmic plot (right $y$-axis, bottom curve) of AFM data for $x=0.4$. Values of $d$ and $L_{s}$ are indicated by arrows. The continuous line indicates the best $\left(W_{\mathrm{AFM}}\right)^{2} \propto \log L$ fitting for $L>L_{s}$. 


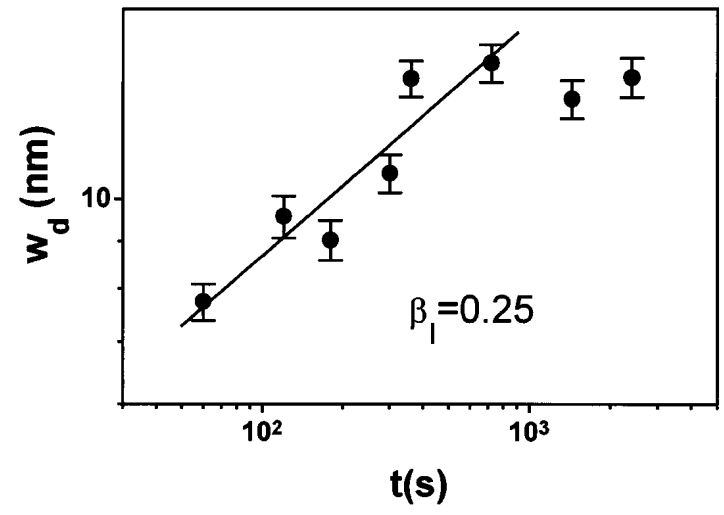

FIG. 5. $W_{d}$ vs $t$ logarithmic plot corresponding to AFM data of copper electrodeposits produced from additive-containing acid plating baths at $j=0.02 \mathrm{~A} \mathrm{~cm}^{-2}$ and $298 \mathrm{~K}$ for $x=0.3$. The straight line is the best $W \propto t^{\beta}$ fitting of the data points before the saturation region, with $\beta=0.25$.

regime is more clearly observed as $x$ increases (Fig. 4). It should be noted that $\alpha=0.77$ and $\beta=0.36$ have been reported for copper electrodeposits on gold grown from thiourea-containing solutions with $x=0.1$ [9], a figure three times smaller than the lowest value of $x$ used in our work. As seen in Figs. 3 and 4(a), both $L^{*}$ and $t_{s}$ increase as $x$ is decreased. Therefore, it would be possible that the asymptotic logarithmic behavior of the interface could not be observed in [9] because of the low value of $x$.

The fact that the EW model describes $\mathrm{Cu}$ electrodeposition from additive-containing plating baths at a low current density in the asymptotic limit suggests that adsorption/desorption is operative. However, it is difficult to understand the contribution of copper adatom desorption from the growing surface under our experimental conditions. Therefore, we propose that the effect of the additive molecules could mimic adsorption/desorption, as occurs when negative barriers at step edges are present [21]. Additive molecules should be preferentially adsorbed at protrusions, where the effect of the electric field becomes greater, hindering copper electrodeposition at these regions. On the other hand, copper electrodeposition should be easier at cavities where the copper surface coverage by the additive molecules should become lower. This fact should result in an enhanced growth at positively curved surfaces as occurs when desorption is operative.

In conclusion, we have found that metal electrodeposition from an additive-containing plating bath can be used as a model system for the evolution of the growing interface obeying the complete linear equation.

This work was financially supported by the Consejo Nacional de Investigaciones Científicas y Técnicas of Argentina (CONICET) and partially realized within the frame of the CONICET-Consejo Superior de Investigaciones Científicas of Spain (CSIC) research cooperation program. L. V. and R. C.S. gratefully acknowledge the
Fundación Ramón Areces for financial support. The authors thank Sandra Mendez for sample preparation.

*Visiting researcher from Instituto de Ciencia de Materiales, CSIC, Cantoblanco, 28049 Madrid, Spain.

[1] P. Meakin, in The Fractal Approach to the Heterogeneous Chemistry, edited by D. Avnir (J. Wiley, New York, 1989), p. 131; M. Matsushita, in The Fractal Approach to the Heterogeneous Chemistry, edited by D. Avnir (J. Wiley, New York, 1989), p. 161.

[2] A. Iwamoto, T. Yoshinobu, and H. Iwasaki, Phys. Rev. Lett. 72, 4025 (1994).

[3] J. M. Pastor and M. A. Rubio, Phys. Rev. Lett. 76, 1848 (1996).

[4] S. F. Edwards and D. R. Wilkinson, Proc. R. Soc. London A 381, 17 (1982).

[5] M. Kardar, G. Parisi, and Y. C. Zhang, Phys. Rev. Lett. 56, 889 (1986).

[6] A.R. Despic, in Comprehensive Treatise of Electrochemistry, edited by B.E. Conway, J. O'M Bockris, E. Yeager, S. U. M. Khan, and R. E. White (Plenum Press, New York, 1983), Vol. 7, p. 451.

[7] R. M. Rynders and R. C. Alkire, J. Electrochem. Soc. 141, 1166 (1994).

[8] M. H. Holzle, C. W. Aspel, T. Will, and D. M. Kolb, J. Electrochem. Soc. 142, 3741 (1995).

[9] W. U. Schmidt, R.C. Alkire, and A.A. Gerwirth, J. Electrochem. Soc. 143, 3122 (1996).

[10] F. Family, Physica (Amsterdam) 168A, 561 (1990), and references therein.

[11] A. Barabasi and E. Stanley, Fractal Concepts in Surface Growth (Cambridge University Press, New York, 1995), and references therein.

[12] J. Villain, J. Phys. I (France) 1, 19 (1991).

[13] D. Wolf and J. Villain, Europhys. Lett. 13, 389 (1990).

[14] J. Krim and G. Palasantzas, Int. J. Modern Phys. B 9, 599 (1995), and references therein.

[15] R. C. Salvarezza, L. Vázquez, H. Míguez, R. Mayoral, C. López, and F. Meseguer, Phys. Rev. Lett. 77, 4572 (1996).

[16] V.G. Levich, Physicochemical Hydrodynamics (Prentice Hall, Englewood Cliffs, NJ, 1962); F. Hine, Electrode Processes and Electrochemical Engineering (Plenum Press, New York, 1985).

[17] L. Vázquez, R. C. Salvarezza, P. Ocón, P. Herrasti, J. M. Vara, and A. J. Arvia, Appl. Sur. Sci. 70/71, 413 (1993).

[18] J. Krim, I. Hevaert, C. Haesendock, and Y. Bruynseraede, Phys. Rev. Lett. 70, 57 (1993).

[19] S. J. Stranick, A. N. Parikh, D. L. Allara, and P. S. Weiss, J. Phys. Chem. 98, 11136 (1994).

[20] J. Krug, in Scale Invariance, Interfaces, and NonEquilibrium Dynamics, edited by A. McKane, M. Droz, J. Vannimenus, and D. Wolf, NATO ASI, Ser. B, Vol. 344 (Plenum Press, New York, 1995), p. 1, and references therein.

[21] S. Majaniemi, T. Ala-Nissila, and J. Krug, Phys. Rev. B 53, 8071 (1996).

[22] C. Alonso, R. C. Salvarezza, J. M. Vara, and A. J. Arvia, Electrochim. Acta 35, 1131 (1990). 